\title{
Genomic analysis of variability in Delta-toxin levels between Staphylococcus aureus strains
}

\author{
Michelle Su ${ }^{1}$, James T Lyles ${ }^{2}$, Robert A Petit III ${ }^{1}$, Jessica M Peterson ${ }^{1}$, Michelle Hargita ${ }^{1}$, Huaqiao Tang ${ }^{2}$, \\ Claudia Solis-Lemus ${ }^{3}$, Cassandra L Quave ${ }^{2,4}$, Timothy D Read ${ }^{\text {Corresp. } 1,3}$ \\ ${ }^{1}$ Division of Infectious Diseases, Department of Medicine, School of Medicine, Emory University, Atlanta, Georgia, United States of America \\ 2 Center for the Study of Human Health, College of Arts and Sciences, Emory University, Atlanta, Georgia, United States of America \\ 3 Department of Human Genetics, School of Medicine, Emory University, Atlanta, Georgia, United States of America \\ 4 Department of Dermatology, School of Medicine, Emory University, Atlanta, Georgia, United States of America \\ Corresponding Author: Timothy D Read \\ Email address: tread@emory.edu
}

Background. The delta-toxin (ס-toxin) of Staphylococcus aureus is the only hemolysin shown to cause mast cell degranulation and is linked to atopic dermatitis, a chronic inflammatory skin disease. We sought to characterize variation in $\delta$-toxin production across $S$. aureus strains and identify genetic loci potentially associated with differences between strains.

Methods. A set of 124 S. aureus strains were genome-sequenced, and $\delta$-toxin levels in stationary phase supernatants determined by high performance liquid chromatography (HPLC). SNPs and kmers were associated with differences in toxin production using four genome-wide association study (GWAS) methods. Transposon mutations in candidate genes were tested for their $\delta$-toxin levels. We constructed XGBoost models to predict toxin production based on genetic loci discovered to be potentially associated with the phenotype.

Results. The $S$. aureus strain set encompassed 40 sequence types (STs) in 23 clonal complexes (CCs). סtoxin production ranged from barely detectable levels to $>90,000$ units, with a median of $>8,000$ units. CC30 had significantly lower levels of and toxin production than average while CC45 and CC121 were higher. MSSA (methicillin sensitive) strains had higher $\delta$-toxin production than MRSA (methicillin resistant) strains. Through multiple GWAS approaches, 45 genes were found to be potentially associated with toxicity. Machine learning models using loci discovered through GWAS as features were able to predict $\delta$-toxin production (as a high/low binary phenotype) with a precision of .875 and specificity of .990 but recall of .333. We discovered that mutants in the carA gene, encoding the small chain of carbamoyl phosphate synthase, completely abolished toxin production and toxicity for Caenorhabditis elegans.

Conclusions. The amount of stationary phase production of the toxin is a strain-specific phenotype likely affected by a complex interaction of number of genes with different levels of effect. We discovered new candidate genes that potentially play a role in modulating production. We report for the first time that the product of the carA gene is necessary for $\delta$-toxin production in USA300. This work lays a foundation for future work on understanding toxin regulation in $S$. aureus and prediction of phenotypes from genomic sequences. 
2 Genomic analysis of variability in Delta-toxin levels

3 between Staphylococcus aureus strains

6 Michelle Su${ }^{1}$, James Lyles ${ }^{2}$, Robert A. Petit III ${ }^{1}$, Jessica M. Peterson ${ }^{1}$, Michelle Hargita ${ }^{1}$,

7 Huaqiao Tang ${ }^{2}$, Claudia Solis-Lemus ${ }^{3}$, Cassandra Quave ${ }^{2,4}$, Timothy D. Read ${ }^{1,3}$

8

$9 \quad{ }^{1}$ Division of Infectious Diseases, Department of Medicine, School of Medicine, Emory

10 University, Atlanta, GA, USA

$11{ }^{2}$ Center for the Study of Human Health, College of Arts and Sciences, Emory University, 12 Atlanta, GA, USA

13 '3epartment of Human Genetics, School of Medicine, Emory University, Atlanta, GA, USA

${ }^{4}$ Department of Dermatology, School of Medicine, Emory University, Atlanta, GA, USA

Corresponding Author:

17 Timothy Read

181760 Haygood Dr NE, Atlanta, GA 30322, USA

19 Email address: tread@emory.edu 


\section{Abstract}

43 Background. The delta-toxin ( $\delta$-toxin) of Staphylococcus aureus is the only hemolysin shown 44 to cause mast cell degranulation and is linked to atopic dermatitis, a chronic inflammatory skin 45 disease. We sought to characterize variation in $\delta$-toxin production across $S$. aureus strains and 46 identify genetic loci potentially associated with differences between strains.

47 Methods. A set of $124 \mathrm{~S}$. aureus strains were genome-sequenced, and $\delta$-toxin levels in 48 stationary phase supernatants determined by high performance liquid chromatography (HPLC). 49 SNPs and kmers were associated with differences in toxin production using four genome-wide 50 association study (GWAS) methods. Transposon mutations in candidate genes were tested for 51 their $\delta$-toxin levels. We constructed XGBoost models to predict toxin production based on 52 genetic loci discovered to be potentially associated with the phenotype.

53 Results. The $S$. aureus strain set encompassed 40 sequence types (STs) in 23 clonal complexes 54 (CCs). $\delta$-toxin production ranged from barely detectable levels to $>90,000$ units, with a median 55

\section{CC45 and CC121 were higher. MSSA (methicillin sensitive) strains had higher $\delta$-toxin} production than MRSA (methicillin resistant) strains. Through multiple GWAS approaches, 45 genes were found to be potentially associated with toxicity. Machine learning models using loci discovered through GWAS as features were able to predict $\delta$-toxin production (as a high/low binary phenotype) with a precision of .875 and specificity of .990 but recall of .333 . We discovered that mutants in the carA gene, encoding the small chain of carbamoyl phosphate synthase, completely abolished toxin production and toxicity for Caenorhabditis elegans.

Conclusions. The amount of stationary phase production of the toxin is a strain-specific phenotype likely affected by a complex interaction of a number of genes with different levels of effect. We discovered new candidate genes that potentially play a role in modulating production. We report for the first time that the product of the car $A$ gene is necessary for $\delta$-toxin production in USA300. This work lays a foundation for future work on understanding toxin regulation in $S$. aureus and prediction of phenotypes from genomic sequences.

\section{Introduction}

Staphylococcus aureus is a common causative agent of nosocomial and community-acquired infections, encoding a wide variety of factors that damage the host and evade immunity. Central to its ability to cause disease is its large repertoire of toxins. S. aureus can produce at least 13 extracellular toxins (Grumann, Nübel \& Bröker, 2014; Otto, 2014; Laabei et al., 2015), including phenol-soluble modulins (PSMs) (Peschel \& Otto, 2013), alpha-toxin (Bhakdi \& Tranum-Jensen, 1991), Panton-Valentine Leukocidin (PVL) (Genestier et al., 2005), and $\delta$-toxin (Wang et al., 2007).

Toxin expression levels are subject to evolutionary trade-offs between survival and transmission . . in different environments (Laabei et al., 2015; Young et al., 2017). Toxins contribute to important biological functions: In S. aureus, alpha-toxin is important for initial cell-to-cell 
82 contacts in biofilm formation, beta-toxin contributes to biofilm structure and growth via 83 crosslinking, and PSMs are involved in detachment of cells for dispersal (Rudkin et al., 2017).

84 In addition, expression of toxins is essential to skin and soft tissue infections and other common 85 diseases caused by the bacterium (Xu \& McCormick, 2012; Otto, 2013; Peschel \& Otto, 2013 ;

86 Kitur et al., 2015). However, during chronic $S$. aureus infections toxin production is a contra-

87 indication of disease as reduced toxicity mutants may have situationally increased fitness

88 (Cheung et al., 2014; Soong et al., 2015; Rose et al., 2015; Laabei et al., 2015). Dysfunction in

89 the Agr quorum sensing system (Novick, 2003), central to upregulation of many toxins, has been 90 linked to longer durations of bacteremia (Fowler et al., 2004; Sakoulas et al., 2005). Similarly, 91 mutational inactivation of another regulator, Rsp, which promotes $S$. aureus infection and 92 93

94

95

96

97

98

99

100

101

102

103

104

105

106

107

108

109

110

111

112

113

114

115

116

117

118

119

120

121

virulence (Li et al., 2015), allows for prolonged survival in chronic infections (Das et al., 2016).

In this study, we focus on the genetics of strain-specific differences of $\delta$-toxin expression. $\delta$ toxin is an amphipathic peptide in the PSM family. It can form pores on the surface of host cells, eliciting a pro-inflammatory response or cytolysis at high concentrations (Bernheimer \& Rudy, 1986; Kasimir et al., 1990; Otto, 2014). $\delta$-toxin is the product of the hld gene, which is part of the Agr quorum sensing system. The Agr operon consists of two divergently transcribed operons $\mathrm{P} 2$ and $\mathrm{P} 3$. The P2 operon encodes the four genes necessary for quorum sensing and activates the P3 operon, which transcribes the main effector of the Agr system, a 514-nucleotide regulatory molecule RNAIII. RNAIII also contains the $h l d$ gene encoding the 26 amino acid $\delta$ toxin peptide, which has been found only in S. aureus and S. epidermidis (McKevitt et al., 1990). In a community-associated MRSA (CA-MRSA) bacteremia mouse model, PSMa and $\delta$-toxin were shown to be important for disease severity, indicating their importance as virulence factors (Wang et al., 2007; Peschel \& Otto, 2013). However, $\delta$-toxin is the only PSM shown to induce mast cell degranulation (Nakamura et al., 2013) and increase the severity of S. aureus of Atopic Dermatitis (AD), a chronic inflammatory skin disease, affecting $15-30 \%$ of children and 5\% of adults in the US and industrialized countries (Williams \& Flohr, 2006; Pustišek, Vurnek Živković \& Šitum, 2016). Despite its importance, we know little of the natural variation in production of the $\delta$-toxin molecule between $S$. aureus strains and the genetic factors that influence this trait. Therefore, we queried the range of $\delta$-toxin production in a diverse set of $S$. aureus strains and attempted to determine if there are genetic loci strongly associated with $\delta$ toxin production by using bacterial genome-wide association study (GWAS) methods. We then analyzed the performance of identified genome variants and metadata for predicting $\delta$-toxin production.

\section{Materials \& Methods}

\section{Strains and growth conditions}

Network on Antimicrobial Resistance in Staphylococcus aureus (NARSA) and Nebraska Transposon Mutant Library (NTML) strains were acquired from BEI resources

(https://www.beiresources.org/) (Table S1). For $\delta$-toxin assays, bacteria were grown on tryptic

Peer] reviewing PDF | (2019:10:41844:2:1:NEW 4 Feb 2020) 
122 soy agar (TSA) plates overnight (18-24 hours) at $37^{\circ} \mathrm{C}$. TSA plates used for NTML strains had

123 the addition of erythromycin $(5 \mu \mathrm{g} / \mathrm{ml})$. Cultures from a single colony were inoculated and

124 grown overnight in tryptic soy broth (TSB) at $37^{\circ} \mathrm{C}$, a $45^{\circ}$ angle, and $200 \mathrm{rpm}$. Final cultures

125 were standardized to a starting cell density of $5 \times 10^{5} \mathrm{CFU} / \mathrm{ml}$ of TSB and grown for 15 hours at

$12637^{\circ} \mathrm{C}$, a $45^{\circ}$ angle, and $275 \mathrm{rpm}$ (Quave \& Horswill, 2018).

127

128

Whole-genome shotgun sequencing

129

DNA extraction and paired-end library prep were performed as manufacturer's instructions

130

(Wizard Genomic DNA Purification Kit, Promega; Nextera XT DNA Library Prep Kit,

131

Illumina). Genome sequencing was performed using both Illumina HiSeq and MiSeq. Raw read

132 data were deposited in the NCBI Short Read Archive under project accession no.

133 PRJNA289526.0. 102/124 strains had more than 40x average genome coverage, and the

134 minimum coverage of any strain was $33 \mathrm{x}$.

135

136

Genome assembly and annotation

137

Genomes were processed using the Staphopia pipeline (Petit \& Read, 2018). BBduk (v37.66)

138 (Bushnell, 2016) was used to eliminate Illumina adapters, trim low quality ends (base quality $<$

139 20), and filter out low quality reads (mean read PHRED quality $<20$ ). Read error correction and de novo genome assembly was performed using SPAdes (v3.11.11) (Bankevich et al.). Genome assemblies were annotated with Prokka (Seemann, 2014) (v1.12) using its default database. SNP-sites was used to call single nucleotide polymorphisms (SNPs) in the core genome alignment with $S$. aureus N315 as the reference strain (Page et al., 2016). Agr type was determined using BLAST to query genome assemblies for the agrD nucleotide sequences of defined agr types: I (AB492152.1), II (AF001782), III (AF001783), and IV (AF288215). For all but four samples, $100 \%$ coverage and $>95 \%$ identity were used to identify Agr type. NRS168, NRS182 NRS235, and NRS260 Agr types were determined based on available metadata. Untyped strains (NA) returned no BLAST results likely due to contig boundaries falling in this region. MLST (multilocus sequencing type) was determined using the SRST2 tool (Inouye et al., 2014) with the PubMLST database (Jolley, Bray \& Maiden, 2018).

151

\section{Phylogenetic tree estimation}

A core genome alignment of 999,473 base-pairs (bp) from the 124 NARSA strains was obtained from Roary (Page et al., 2015; Tange, 2018) (v3.11.2). Gubbins was used to remove potential recombination regions and to obtain a downsized core genome alignment of 42,406 bp containing only polymorphic sites (Croucher et al., 2015). A final maximum likelihood (ML) tree was obtained with RAxML (v8.2.10) with 100 bootstraps and a GTRGAMMA model

158 (Stamatakis, 2014).

159

\section{Toxin identification using HPLC}


161 High performance liquid chromatography (HPLC) methods was employed to detect and quantify

162

163

164

165

166

167

168

169

170

171

172

173

174

175

176

177

178

179

180

181

182

183

184

185

186

187

188

189

190

191

192

193

194

195

196

197

198

199

200

the levels of $\delta$-toxin present in supernatants of 124 NARSA strains following established procedures (Quave \& Horswill, 2018). Briefly, a $1.5 \mathrm{ml} \mathrm{S}$. aureus culture grown for 15 hours as described above was centrifuged, and the supernatant transferred to an HPLC vial and frozen at $20^{\circ} \mathrm{C}$ until ready for HPLC testing. HPLC was performed with the following parameters: $500 \mu \mathrm{l}$ injection, flow rate of $2 \mathrm{~mL} / \mathrm{min}$, and UV/Vis monitored at $214 \mathrm{~nm}$ using solvents (a) $0.1 \%$ (vol/vol) trifluoracetic acid in water and (b) $0.1 \%$ trifluoracetic acid in acetonitrile. Peaks at retention time $\sim 7.2 \mathrm{~min}$ and $\sim 7.5 \mathrm{~min}$ corresponding to deformylated and formylated $\delta$-toxin respectively, were quantified by taking the sum of the total peak area. Peak areas were normalized using $\mathrm{OD}_{600}$ readings of the cultures. Prior studies using this HPLC method confirmed peak identity at these retention by LC-MS (Somerville et al., 2003; Quave, Plano \& Bennett, 2011). Analyses were performed on three replicate supernatants per strain.

For subsequent analyses treating toxin production as a continuous variable, we used a Box-Cox power transformation to achieve a more symmetric distribution, and thus, satisfy the normality assumption of phylogenetic regression and other comparative models. For analyses treating toxin production as a binary Low/High, we used a cutoff of 20,000, which clustered strains on the left-side of the distribution and split the data into 109 low and 15 high toxin producers.

\section{Hemolysis assay}

TSA with 5\% rabbit's blood were used to test transposon strains for reduced hemolysis as rabbit blood is more susceptible to $\delta$-toxin. TSA II with 5\% sheep's blood agar were used to test the hemolysis profile of complemented strains. Strains were spotted and incubated at $37^{\circ} \mathrm{C}$ for 24 hours before incubation at $4^{\circ} \mathrm{C}$ for an additional 24 hours. Photos of the plates were taken with the use of a lightbox to illuminate hemolysis zones. Images were imported into software ImageJ (Schneider, Rasband \& Eliceiri, 2012) to increase the contrast of the image and for measurement of hemolysis zones by taking the hemolysis measurement and subtracting colony size.

\section{GWAS}

All GWAS analyses were done with $106 \mathrm{~S}$. aureus strains. NRS168, NRS252-NRS256, NRS259, NRS260, NRS262, NRS264-NRS266, NRS271, NRS272, NRS275, NRS383, NRS386, NRS387, and NRS408 were later phenotyped and included in all other analyses.

\section{SEER (Lees et al., 2016) (v 1.1.4) was run according to} https://github.com/johnlees/seer/wiki/Tutorial. In brief, kmers used for SEER were counted using fsm-lite (https://github.com/nvalimak/fsm-lite) using genome assemblies in fasta format as input. Population structure was estimated using Mash (Ondov et al., 2016) to sketch assemblies and output pairwise distances between all samples. SEER scripts were used to create a distance matrix. Six dimensions was chosen based on scree plot output (Fig. S1). SEER was run using a binary phenotype with filtering $\mathrm{p}$ value filtering off. QQ plots were made in $\mathrm{R}$ to ensure that

Peer] reviewing PDF | (2019:10:41844:2:1:NEW 4 Feb 2020) 
201 population structure was properly accounted for. A minor allele frequency (MAF) of .20 was 202 chosen due as regression analysis with kmers of lower MAF tend to fail or have high standard 203 errors. Significant kmers were kmers with likelihood ratio test p-values lower and equal to the 204 Bonferroni correction of .05/n, where $\mathrm{n}$ is the number of kmers tested. Significant kmers were 205 mapped to reference genome N315 (NC_002745.2) using BLAST (Camacho et al., 2009) 206 optimized for short queries. Bedtools (v2.27.1) (Quinlan \& Hall, 2010) was then used to 207 annotate the matches.

208

For treeWAS (Collins \& Didelot, 2017), a binary core SNPs matrix was generated from snp-sites

210 output. To account for the right skewed distribution of $\delta$-toxin production, the values were transformed into ranks. treeWAS was run with 3 unrooted trees (NARSA strains alone, NARSA strains plus ST93, NARSA strains plus $S$. argenteus) generated from RAxML (v8.2.10) to limit false positives generated from an incorrect phylogeny, and the union of all loci identified were considered significant.

215

For bugwas (Earle et al., 2016), we used a modified version of GEMMA 0.93 (Zhou \& Stephens, 2012), with a centered relatedness matrix (GEMMA option -gk 1) created using BIMBAM files and a binary toxin phenotype file and set Minor Allele Frequency of 0. Biallelic core SNPs were used to create a mean genotype file, and SNP positions were noted in a SNP annotation file with the chromosome number set to 24 to indicate one allele. A nucleotide matrix of core SNPs, a binary phenotype file, and an unrooted phylogenetic tree created by RAxML (v8.2.10) were used to run the bugwas R package. A Bonferroni correction of $.05 / \mathrm{n}$, where $\mathrm{n}$ was the sum of phylogenetic patterns represented by the bi and tri allelic SNPs.

DBGWAS (Jaillard et al., 2018) (v0.5.0) was run using genome assemblies in fasta format, a binary phenotype, unrooted phylogenetic tree created by RAxML (v8.2.10), and DBGWAS resistance and UniProt databases for annotation. A false discovery rate (FDR) of 5\% was used to

229

230

231

232

233

234

235 determine significant kmers.

\section{Phylogenetic Regression}

We fit three phylogenetic comparative models: 1) a phylogenetic regression to study the association of $\delta$-toxin production and several covariates, 2) a Pagel's lambda model to estimate the phylogenetic signal of $\delta$-toxin production, and 3) an ancestral state reconstruction to $\delta$-toxin production along the branches of the Staphylococcus aureus phylogeny.

For the phylogenetic regression model, we include $\delta$-toxin level as continuous response for 106 238 strains and 11 predictors: clonal complex (CC), methicillin-resistant S. aureus (MRSA), Agr type (agr), and variants associated with $\delta$-toxin production in $S$. aureus identified using SEER (Lees et al., 2016) and bugwas (Earle et al., 2016) and DBGWAS (Jaillard et al., 2018) (Table S3). isdC and WP 000894032.1 were excluded from the analysis due to large standard errors while 
241 sequence type (ST) was excluded due to similarity to CC. Bugwas variants were represented in 242 the analysis as 6 phylogenetic patterns. We used the julia package PhyloNetworks (Solís-Lemus,

243 Bastide \& Ané, 2017; Bastide et al., 2018) to fit the phylogenetic regression, to estimate the

244 phylogenetic signal in $\delta$-toxin through Pagel's $\lambda$ transformation, and to reconstruct the ancestral

245 states. To tease apart which if any of these factors truly impact $\delta$-toxin production, we

246 performed a phylogenetic regression. First, with Pagel's lambda model, we estimated a strong

247 phylogenetic signal $(\lambda=.504035)$ for the $\delta$-toxin production using a rooted (by $S$. argenteus)

248 phylogenetic tree calibrated to be consistent with time. This estimate did not entirely fit under

249 the Brownian Model (BM) assumption, which requires $\lambda \approx 1$. Regardless of this, we assumed a

$250 \mathrm{BM}$ for the evolution of $\delta$-toxin production in the phylogenetic regression model. The rationale

251 for the use of BM was its simplicity, as well as the shown robustness to model misspecification

252 (Bastide et al., 2018).

253

254

255

\section{Extreme Gradient Boosted Tree Classifier}

The R package xgboost (Chen \& Guestrin, 2016) was used to create predictive classifiers with

256

257

258

259

260

261

262

263

264

265

266

267

268

269

270

271

272

273

274

275

276

277

278

279

280 strain metadata and genetic features from the GWAS. The predictor was trained using stratified 10 -fold cross-validation wherein $90 \%$ is used for training and $10 \%$ is used for validation. Model performance metrics such Area Under Receiver Operating Characteric (AUROC) and Cohen's Kappa were calculated using R packages pROC and irr respectively.

\section{Other Statistical Analysis}

Association of MRSA/MSSA status, Agr type, and CC to toxin production was performed with Kruskall-Wallis and pairwise Mann-Whitney $U$ tests with continuous $\delta$-toxin used as the dependent variable. A Bonferroni correction was applied to test $\mathrm{p}$-values to account for multiple tests. Pagel's lambda and Blomberg's K (Pagel, 1999; Blomberg, Garland \& Ives, 2003) were estimated using R package phytools using an unrooted phylogenetic tree obtained from RAxML and no calibration to the branch lengths. All analysis was performed using the $\mathrm{R}$ (R Core Team, R Foundation for Statistical Computing, Vienna, Austria., 2016) and Julia programming language (Bezanson et al., 2017) for statistical computing.

\section{Phenotypic analysis of toxin phenotypes of transposon mutant strains}

Nine transposon mutants in genes potentially associated with $\delta$-toxin production were selected from the USA300 Nebraska transposon library (Fey et al., 2013). In addition, we selected an agrA mutant as a positive control for $\delta$-toxin disruption and one randomly chosen mutant with no known association from the GWAS experiments as a negative control. The gene disrupted was a phi77 ORF109-like protein, SAUSA300_1928, WP_000582165.1. All transposon mutants were transduced into an isogenic USA300 JE2 background and confirmed by PCR (Table S2). HPLC assays and hemolysis assays for $\delta$-toxin were as previously described. Complementation was performed by cloning PCR fragments containing the USA300 genes into the pOS1-Plgt vector using splicing overlap extension PCR (Bubeck Wardenburg, Patel \& Schneewind, 2007). In 
281 brief, the plasmid and genes were PCR amplified to contain complementary overhangs. The 282 purified products were then mixed and subject to another round of PCR with no primers. This 283 reaction was used to transform IM08B E. coli (Monk et al., 2015). The plasmid was purified and 284 electroporated into the mutant strains as previously described (Monk et al., 2012). A pOS1-Plgt 285 only plasmid was used as a control for complementation experiments. The C. elegans virulence 286 assays were performed using C. elegans strain N2. Nematode population synchronization was 287 performed as in Penley et al (Penley \& Morran, 2018). Populations were bleached in $20 \%$ 288 household bleach and M9 buffer and plated on OP50 until L4 larval stage (48 hours at $20^{\circ} \mathrm{C}$ ).

289

290

291

292

293

294

295

296

297

298

299

300

301

302

303

304

305

306

307

308

309

310

311

312

313

314

315

316

317

318

319

320 Worms were subsequently washed off, counted, and $\sim 200$ were plated on control OP50 plates and S. aureus lawns on BHI agar. S. aureus plates were prepared 24 hours prior by adding 200 ul of an overnight culture and growing at $24{ }^{\circ} \mathrm{C}$. At 24 hours and 48 hours, plates were scored by counting live worms. Worm counts on OP50 plates were used to normalize mortality calculations and to account for plating efficiency.

\section{Results}

$\delta$-toxin production level is highly variable between $S$. aureus strains and is associated with MSSA/MRSA and Clonal Complex

We used high performance liquid chromatography (HPLC) to quantify stationary phase $\delta$-toxin production in 124 publically available $S$. aureus strains from the Network on Antimicrobial Resistance in Staphylococcus aureus (NARSA) collection, which represent diverse taxonomic groups within the species (Table S1). The strains, which were shotgun sequenced using Illumina technology, were a diverse representation of the $S$. aureus species, consisting of 40 sequence types (STs) in 23 clonal complexes (CCs). There was considerable variation in the total $\delta$-toxin production (sum of the formylated and deformylated $\delta$-toxin peptides) between strains (Fig. 1, Fig. S2). The distribution most closely fits a gamma model with a strong left skew. Production ranged from zero to 97,235 units, with a median value of 8,295 . The majority of strains produced less than 10,000 units; 118 of the 124 strains (95\%) produced less than 30,000.

When toxin production was mapped onto the strain phylogeny, it was apparent that there was variation in the average level between clonal complexes even though there was also a large variation in the phenotype within CCs (Fig. 2). Two tests for the phylogenetic signal of the trait, Blomberg's K and Pagel's lambda (Pagel, 1999; Blomberg, Garland \& Ives, 2003), returned statistically robust scores $(\mathrm{K}=.019, \mathrm{p}=.016 / \lambda=.99, \mathrm{p}=1.55 \mathrm{e}-48$; for both measures, a value of 1 indicate trait similarity measured by variance $(K)$ or correlation $(\lambda)$ as expected under Brownian evolution). A Pagel's lambda value of $\sim 1$ indicates a strong phylogenetic signal, while the low Blomberg's $\mathrm{K}$ indicates that the variance that exists within $\delta$-toxin production is primarily on the tips of the trees within clades and does not wholly fit a Brownian model of evolution for $\delta$-toxin. When variation between clades was analyzed pair-wise using KruskalWallis and Mann-Whitney tests, CC30 (Average $\delta$-toxin 4299) was found to have significantly lower $\delta$-toxin production than CC45 (Average $\delta$-toxin 30955, $\mathrm{p}=.027$ ) and CC121 (Average $\delta$ - 
321

322

323

324

325

326

327

328

329

330

331

332

333

334

335

336

337

338

339

340

341

342

343

344

345

346

347

348

349

350

351

352

353

354

355

356

357

358

359

360

361

toxin $17693, p=.00042$, Fig. 3c). Ancestral reconstruction of the $\delta$-toxin phenotype (Fig. S3) suggested that high $\delta$-toxin producing clades such as CC45, CC890 and CC72 had arisen independently in the $S$. aureus species from a low producing ancestor.

Agr groups have been suggested to be associated with differences in $S$. aureus cytotoxicity (Jarraud et al., 2002; Collins, Buckling \& Massey, 2008). All four Agr groups were present in our samples (I: 50, II: 22, III: 38, IV: 11, NA: 3). We found significant differences in toxin between Agr I and III ( $\mathrm{p}=.022)$ using Kruskal-Wallis test and between Agr III and IV ( $\mathrm{p}=$ .00049) using pairwise Mann-Whitney U tests. Agr I and IV have higher mean levels than Agr II and III (Fig. 3b). Methicillin resistance has also been previously indicated to interfere with the Agr quorum sensing system and thus toxin production (Rudkin et al., 2012). Within our set of strains, MRSA strains were found to have lower $\delta$-toxin than MSSA strains by Mann-Whitney $U$ $(p=0.024$, Fig. 3a). Some caution must be used when assigning causality as clonal complex, Agr group and MRSA status are strongly confounded, but we also found that MRSA status and Agr type III were significant negative predictors of $\delta$-toxin level in a phylogenetic regression (see methods).

\section{Diverse genetic loci are associated with variation in individual strain $\delta$-toxin production levels}

To ascertain individual genomic variants that may potentially be associated with the differences in toxin production, we used four recently published programs for bacterial GWAS (genomewide association studies). The programs differ in the population structure correction, the types variants tested and whether continuous or binary phenotypes could be tested. SEER (Lees et al., 2016) is an alignment-free method that uses kmers as features to create a distance matrix and a fixed effects model to correct for population structure. SEER, therefore allows discovery of both core and accessory gene variants associated with the phenotype. For the purposes of GWAS, we defined the binary toxin phenotype at a cutoff of 20,000 units (Fig. 1), which gave a set of 87 strains in the "low" toxin and 19 in the high toxin category. This threshold was chosen to separate the very high-producing strains from the main mass (Fig. 1). Using the binary phenotype, SEER identified three genes having more than ten kmers with statistically significant association (Table S3). Polymorphisms in $i s d C$, $g l p D$, and a gene encoding YbbR-like domaincontaining protein (WP000894032.1) were found to be negatively associated with $\delta$-toxin production. Bugwas (Earle et al., 2016) is an alternative distance-based GWAS program that uses principal components as fixed effects for population structure control. Bugwas analysis, performed with a binary phenotype, produced six phylogenetic patterns of SNPs (Table S3). DBGWAS (Jaillard et al., 2018) is a kmer based alignment-free method which relies on De Brujin graphs to interpret genomic variation but uses bugwas for downstream analyses. Running DBGWAS with a binary phenotype yielded one hit in an intergenic region near staphopain A (Table S3). treeWAS (Collins \& Didelot, 2017) differed from the other three programs in using the strain phylogeny to model changes associated with phenotype changes at the tip and within the structure of the tree. Using a phylogenetic tree can be more accurate than distance estimation 
362 if the tree is correct, therefore for robustness we used three separate trees (NARSA strains only,

363

364

365

366

367

368

369

370

371

372

373

374

375

376

377

378

379

380

381

382

383

384

385

386

387

388

389

390

391

392

393

394

395

396

397

398

399

400

401

402

NARSA strains with a ST93 outgroup, NARSA strains with an S. argenteus outgroup) and pooled common loci. Using a ranked phenotype, genes common to all three analyses were $f a d D$, $v r a D, \operatorname{deg} A, g d p P, g g t, s u f B, o p c R, r e b M$, thiD, and three uncharacterized proteins (Table S3).

When the results from the four approaches were aggregated (Table S3), we noted the majority of the genetic loci were in genes encoding enzymes that were part of conserved metabolic pathways. Forty-two variants were synonymous mutations, and only nine were nonsynonymous (6 loci from the bugwas analysis and 3 from treeWAS). None of the four GWAS approaches found any significant genetic loci in common.

We attempted to determine if machine learning approaches could predict $\delta$-toxin from genome sequences by integrating information from the diverse GWAS analyses with MRSA/MSSA, Agr group and genotype (CC or ST). We chose Extreme Gradient Boosting (XGBoost) (Friedman, 2001; Chen \& Guestrin, 2016) which uses decision tree ensembles to predict from the given set of features. XGBoost has been used to successfully predict biologically relevant phenotypes such as antibiotic resistance in Enterobactericiae and Salmonella (Nguyen et al., 2018, 2019) as well as RNA-protein interactions (Jain, Gupte \& Aduri, 2018), protein-protein interactions (Wang, Liu \& Deng, 2018; Sanchez-Garcia et al., 2019), and RNA methylation (Qiang et al., 2018). An XGBoost model was trained with stratified 10-fold cross-validation. When using a binary $\delta$-toxin phenotype (> 20000), the model had a precision of .875 and a recall of .333 . Specificity was .990, and the Area Under Receiver Operating Characteristic (AUROC) was .697 (Table 1). Interestingly, excluding MRSA/MSSA status, Agr group and ST/CC had no effect on model performance, suggesting that rare genomic variants are the main driving force of very high $\delta$-toxin production. Recall was poor, suggesting there are yet unfound genomic determinants that contribute strongly to the phenotype. Splitting the toxin levels into 4 categories $(0-1000,1001-7000,7001-30000,>30000)$ decreased performance (With metadata: .423 weighted precision, .443 weighted recall, .664 AUROC; Without metadata: .451 weighted precision, .326 weighted recall, .667 AUROC). However, most of the errors in the 4-category model ( $75 \%)$ occurred in adjacent categories, suggesting that the classifier was better than random choice with near-misses. ST was the top parameter in prediction, resulting in a loss of $\sim 21 \%$ accuracy when omitted. This suggested that the driving force behind differential $\delta$-toxin level in $S$. aureus is interactions between a potentially large number of genes with the potential to affect toxin expression levels.

\section{The small chain of carbamoyl phosphate synthase, encoded by $\operatorname{car} A$, is necessary for $\delta$-toxin production in USA300}

We screened the $\delta$-toxin production phenotype of transposon mutants of 9 of the 42 genes putatively identified by GWAS (Table S3) as well as agrA as a positive control and a randomly chosen gene as negative control (SAUSA300_1928). We used mutants from the USA300

Nebraska Transposon Mutant Library (Fey et al., 2013) that were transduced back into the

Peer] reviewing PDF | (2019:10:41844:2:1:NEW 4 Feb 2020) 
403 parental USA300 JE2 strains and validated by PCR (Table S2). Of the eleven mutants tested, 404 transposons in hemL, carA, $g l p D$, is $d C$, thiD, and agrA significantly reduced $\delta$-toxin production 405 (Fig. 4), but only carA, agrA, and isdC mutants showed significantly different hemolysis on 406 rabbit blood agar by Mann Whitney $U$. $\delta$-toxin production in strains containing transposons in $407 f a d D, s b n C, b r n Q, h \lg B$, and phi77 ORF109-like protein was not different to the parental strain 408 USA300 JE2. agrA is necessary to activate RNAIII and hld transcription (Janzon, Löfdahl \& 409 Arvidson, 1989; Gagnaire et al., 2012), so the transposon knock-out was expected to completely 410 abrogate expression. However, the complete shutdown of toxin expression in the car $A$ mutant 411 had not been previously reported. We showed that $\delta$-toxin accumulation by the car $A$ mutant 412 could be rescued by a cloned version of the gene on an expression plasmid $(71 \% \delta$-toxin 413 production restoration compared to USA300 JE2) but not an empty vector $(0 \% \delta$-toxin 414 production restoration). car $A$ encodes the carbamoyl-phosphate synthase small chain protein, 415 which is involved in L-arginine biosynthesis and UMP biosynthesis (part of pyrimidine 416 metabolism) and has been shown to potentially regulate nitric oxide resistance (Grosser et al., 417 2018) and be important for the regulation of PSM $\alpha 1$ expression (Hardy et al., 2019). We 418 confirmed the results of Bae et al (Bae et al., 2004) that a carA mutant was defective in killing 419 Caenorhabditis elegans and showed this phenotype could be restored by complementation.

420

421

422

423

424

425

426

427

428

429

430

431

432

433

434

435

436

437

438

439

440

441

442 While hemolysis of the complementation strain on sheep blood agar was less than the original strain (Table 2, Fig. 5a-e), virulence in C. elegans was similar. Additionally, complementation with a car $A$ allele from a CC45 high $\delta$-toxin strain that restored hemolysis to comparable levels, but not $\delta$-toxin production showed reduced killing of $C$. elegans (Fig. 5f). This result suggests that $\delta$-toxin may play an important role in virulence in this model organism.

\section{Discussion}

This study revealed the complex relationship between strain phylogeny and $\delta$-toxin accumulation at stationary phase in $S$. aureus. The phenotype had a strikingly left-skewed distribution, with a minority of strains having $>5$-fold the median value in toxin units (Fig. 1). Pathoadaptation probably plays a major role is generating this diversity: $\delta$-toxin has been shown to be an important virulence factor in skin and soft tissue infection (Berlon et al., 2015), but in bacteremia agr-regulated toxins may be under negative selection (Fowler et al., 2004; Sakoulas et al., 2005). Toxin expression levels may also change through neutral genetic variation or selection on other metabolic pathways, especially if the levels of the toxin are ultimately determined by the interaction of multiple complex regulatory pathways (Priest et al., 2012). We showed there was a strong relationship between phylogeny and $\delta$-toxin expression (Fig. 3c). Ancestral reconstruction of $\delta$-toxin levels (Fig. S3) suggested higher expression has evolved several times independently but in a minority of clades, indicative of the fitness trade-offs that can exist with increased virulence. Strains also vary considerably within CCs, suggesting within-clade mutations affect the level of expression. An example of this is the NRS22 strain in CC45, which had a more 4-fold less production than the average for the CC (NRS22 =6,686; CC45 average = 30,955) (Fig. 2). The association of higher or lower levels with particular CCs is likely due to 
443 epistatic interaction between rare mutations and variants shared between clade members. As the 444 strains in this study originate from a wide range of infections (Table S1), it was not possible to

445

446

447

448

449

450

451

452

453

454

455

456

457

458

459

460

461

462

463

464

465

466

467

468

469

470

471

472

473

474

475

476

477

478

479

480

481

482 associate $\delta$-toxin production in $S$. aureus with a particular disease (such as atopic dermatitis).

We used multiple bacterial GWAS approaches to produce a list of candidate loci that may be affecting $\delta$-toxin production at different phylogenetic levels. GWAS looks for homoplasic genetic variants produced by recombination or parallel evolution that can be associated with phenotypic variation (Read \& Massey, 2014; Power, Parkhill \& de Oliveira, 2016). Methods vary by the types of variant tested (SNPs, kmers, indels), whether continuous or discrete phenotypes are used and methods for controlling non-independence of samples due to shared ancestry and typically widespread linkage disequilibrium. Two main approaches have been implemented to determine the underlying population structure of tested bacteria. The most common is a form of principal components correction whereby the genomes of strains are used to create a matrix of linearly uncorrelated variables which can then be included in as either fixed or random effects in a regression model (e.g DBWAS, bugwas, SEER). The second approach uses a phylogenetic tree as input to determine genetic relatedness between strains and can be fed into a regression model as with principal components or used to simulate null data to determine a cut-off for true associations (e.g treeWAS). Although all the variants listed in Table S3 passed the family-wise correction cutoff by their respective programs, many are likely false positives due to the presence of linkage disequilibrium or from underestimation of the underlying population structure, especially since the majority were synonymous substitutions. SEER and treeWAS appeared to be finding SNPs that were common in the S. aureus population (in $\sim 1500$ 20,000 of the $\sim 44,000$ strains in the Staphopia database (Petit \& Read, 2018)). In contrast, bugwas found SNPs strongly associated with CC45 (the most toxic CC), while DBGWAS found a variant associated with low toxin production that excludes all high toxin strains in CC45. Of note, mecA kmers were not discovered by GWAS methods, although phylogenetic regression pointed to MSSA strains having higher toxicity. Similarly, we didn't find any variants within agr genes associated with differences in the phenotype, possibly because they were too rare in our population. Although, it is possible to use GWAS to find novel SNPs with large effect sizes using a relatively small number of genomes within one CC (Laabei et al., 2014), the conclusion to be drawn from this pilot study is that larger numbers of $S$. aureus genomes will be needed for the $\delta$-toxin phenotype across multiple CCs.

Two other GWAS studies have focused on toxicity in S. aureus. Laabei et al were able to build a random forest predictor using 31 SNPs and 21 indels to predict low, medium, and high toxicity in MRSA with an accuracy of $>85 \%$ (Laabei et al., 2014). Recker et al (Recker et al., 2017) clarified the role of toxicity by determining factors associated with bacteremia-associated mortality. Five genes, including two in the agr operon, were selected by random forest to be predictive of mortality in CC22 and CC30 S. aureus bacteremia. None of the list of genes putatively associated with variation in toxin production from these two studies overlap ours, 
483 although our work differed in having a focus specifically on $\delta$-toxin. Genes from our list that 484 have been implicated in other work are hemL1, which is part of the agrA transcriptional pathway 485 (Das et al., 2016; Young et al., 2017) and brnQ. Other genes found to have reduced rabbit blood 486 hemolysis that did not overlap our set were hemB, qox $A-C$ and $h \lg A$. Our GWAS results 487 suggested variants in qoxD, hem $C$ and $h \lg B$ affected in delta toxin production but these have not 488 been shown to have any toxin-related phenotype in any study we have seen. Mutations in $\operatorname{clp} X$ 489 and walK, which were found in our GWAS results, have also been shown to affect hemolysis 490 (Frees et al., 2003; Delauné et al., 2012; Jacquet et al., 2019).

491

492

493

494

495

496

497

498

499

500

501

502

503

504

505

506

507

508

509

510

511

512

513

514

515

516

517

518

519

520

521

522

We validated 9 candidate genes for the effect that transposon mutations had on $\delta$-toxin production. Some candidate genes essential for cellular survival (e.g. $\operatorname{clp} X$ and walK) cannot be tested using knockout mutants. Transposons in 5 genes (two predicted by bugwas, two by SEER and one using treeWAS) had no effect on production, indicating they were likely false positive calls. The finding that hemL, $g l p D$, $i s d C$, and thiD knockouts resulted in a small but significant reduction $\delta$-toxin levels suggests that they may have a significant functional role. The USA300 car $A$ knockout had the most dramatic phenotype as the gene was found to be indispensable for $\delta$ toxin production, a result not previously reported. A non- $\delta$-toxin producing car $A$ mutant was found to have reduced virulence in $C$. elegans, suggesting a role for $\delta$-toxin in infection. Further mechanistic studies are now needed to understand why car $A$ is necessary. Strikingly, the variant discovered through the GWAS screen, and all mutations in the gene in high production CCs such as CC45 were synonymous. There is a growing body of literature documenting differences in protein function caused by synonymous mutations that impact RNA toxicity (Mittal et al., 2018) or protein folding (Walsh, Bowman \& Clark, 2019). Given the involvement of the Agr system, there is possibly a role for mutations to change RNAIII binding specificity and influence gene regulation.

This work (and other GWAS studies) suggest that strain-to-strain variation in $\delta$-toxin production is governed by complex genetic interactions. The high number of significant but probably low effect genes discovered in this analysis highlights the complex regulation of the $\delta$-toxin phenotype and may parallel models proposed for the genetic basis of some traits in eukaryotes (Boyle, Li \& Pritchard, 2017). Nevertheless, we showed that we can train a classifier (XGBoost) using only genome features with prediction accuracies of $87.9 \%$ (binary categories) and $43.5 \%$ (4 categories). We found that the most important predictive feature was ST in the non-binary model, which reflects how much of the variation in $\delta$-toxin production between strains is dependent on phylogeny. The ability to predict phenotypes of toxicity based on sequence data is likely to become an important diagnostic tool as medicine increasingly adopts genome-based technologies (Laabei et al., 2014). Going forward, we can improve genome-based predictors and gain mechanistic insights that may lead to development of anti-toxin drugs through a combination of efforts to expand collection of phenotypic variation in natural strains and molecular genetic studies targeted at high-effect loci. 
523

524

525

526

527

528

529

530

531

532

533

534

535

536

537

538

539

540

541

542

543

544

545

546

547

548

549

550

551

552

553

554

555

556

557

558

559

560

561

562

\section{Conclusions}

$\delta$-toxin production in S. aureus is a strain-specific phenotype likely affected by a complex network of genes. GWAS and machine learning approaches have proved successful in determining genetic determinants underlying this phenotype and using them for genome-based prediction. While most genes discovered by GWAS modify $\delta$-toxin production, car $A$ was found to be essential. Differences in car $A$ function may contribute to virulence by modulating $\delta$-toxin production. Further studies are needed to understand toxin regulation in $S$. aureus and to predict phenotypes from genomic sequences.

\section{Acknowledgements}

We would like to thank Levi Morran and McKenna Penley for help with C. elegans experiments and John Lees for discussion about running GWAS analysis. The discussed strains were provided by the Network on Antimicrobial Resistance in Staphylococcus aureus (NARSA) for distribution by BEI Resources, NIAID, NIH: Staphylococcus aureus.

\section{References}

Bae T, Banger AK, Wallace A, Glass EM, Aslund F, Schneewind O, Missiakas DM. 2004. Staphylococcus aureus virulence genes identified by bursa aurealis mutagenesis and nematode killing. Proceedings of the National Academy of Sciences of the United States of America 101:12312-12317. DOI: 10.1073/pnas.0404728101.

Bankevich A, Nurk S, Antipov D, Gurevich AA, Dvorkin M, Kulikov AS. 498 Lesin VM, Nikolenko SI, Pham S, Prjibelski AD, Pyshkin AV, Sirotkin AV, 499 Vyahhi N, Tesler G, Alekseyev MA, Pevzner PA. 2012. SPAdes: A new 500 genome assembly algorithm and its applications to single-cell sequencing. The Journal 501:455-477.

Bastide P, Solís-Lemus C, Kriebel R, William Sparks K, Ané C. 2018. Phylogenetic Comparative Methods on Phylogenetic Networks with Reticulations. Systematic biology 67:800-820. DOI: 10.1093/sysbio/syy033.

Berlon NR, Qi R, Sharma-Kuinkel BK, Joo H-S, Park LP, George D, Thaden JT, Messina JA, Maskarinec SA, Mueller-Premru M, Athan E, Tattevin P, Pericas JM, Woods CW, Otto M, Fowler VG Jr. 2015. Clinical MRSA isolates from skin and soft tissue infections show increased in vitro production of phenol soluble modulins. The Journal of infection 71:447457. DOI: 10.1016/j.jinf.2015.06.005.

Bernheimer AW, Rudy B. 1986. Interactions between membranes and cytolytic peptides. Biochimica et biophysica acta 864:123-141.

Bezanson J, Edelman A, Karpinski S, Shah V. 2017. Julia: A Fresh Approach to Numerical Computing. SIAM Review 59:65-98. DOI: 10.1137/141000671.

Bhakdi S, Tranum-Jensen J. 1991. Alpha-toxin of Staphylococcus aureus. Microbiological reviews 55:733-751. 
563 Blomberg SP, Garland T Jr, Ives AR. 2003. Testing for phylogenetic signal in comparative data:

564

565

566

567

568

569

570

571

572

573

574

575

576

577

578

579

580

581

582

583

584

585

586

587

588

589

590

591

592

593

594

595

596

597

598

599

600

601

602 behavioral traits are more labile. Evolution; international journal of organic evolution 57:717-745.

Boyle EA, Li YI, Pritchard JK. 2017. An Expanded View of Complex Traits: From Polygenic to Omnigenic. Cell 169:1177-1186. DOI: 10.1016/j.cell.2017.05.038.

Bubeck Wardenburg J, Patel RJ, Schneewind O. 2007. Surface proteins and exotoxins are required for the pathogenesis of Staphylococcus aureus pneumonia. Infection and immunity 75:1040-1044. DOI: 10.1128/IAI.01313-06.

Bushnell B. 2016. BBMap short read aligner. University of California, Berkeley, California. URL http://sourceforge. net/projects/bbmap.

Camacho C, Coulouris G, Avagyan V, Ma N, Papadopoulos J, Bealer K, Madden TL. 2009. BLAST+: architecture and applications. BMC bioinformatics 10:421. DOI: 10.1186/14712105-10-421.

Chen T, Guestrin C. 2016. XGBoost: A Scalable Tree Boosting System. In: Proceedings of the 22Nd ACM SIGKDD International Conference on Knowledge Discovery and Data Mining. KDD '16. New York, NY, USA: ACM, 785-794. DOI: 10.1145/2939672.2939785.

Cheung GYC, Kretschmer D, Duong AC, Yeh AJ, Ho TV, Chen Y, Joo H-S, Kreiswirth BN, Peschel A, Otto M. 2014. Production of an attenuated phenol-soluble modulin variant unique to the MRSA clonal complex 30 increases severity of bloodstream infection. PLoS pathogens 10:e1004298. DOI: 10.1371/journal.ppat.1004298.

Collins J, Buckling A, Massey RC. 2008. Identification of factors contributing to T-cell toxicity of Staphylococcus aureus clinical isolates. Journal of clinical microbiology 46:2112-2114. DOI: $10.1128 / J C M .00156-08$.

Collins C, Didelot X. 2017. A Phylogenetic Method To Perform Genome-Wide Association Studies In Microbes That Accounts For Population Structure And Recombination. bioRxiv:140798. DOI: 10.1101/140798.

Croucher NJ, Page AJ, Connor TR, Delaney AJ, Keane JA, Bentley SD, Parkhill J, Harris SR. 2015. Rapid phylogenetic analysis of large samples of recombinant bacterial whole genome sequences using Gubbins. Nucleic acids research 43:e15. DOI: 10.1093/nar/gku1196.

Das S, Lindemann C, Young BC, Muller J, Österreich B, Ternette N, Winkler A-C, Paprotka K, Reinhardt R, Förstner KU, Allen E, Flaxman A, Yamaguchi Y, Rollier CS, van Diemen P, Blättner S, Remmele CW, Selle M, Dittrich M, Müller T, Vogel J, Ohlsen K, Crook DW, Massey R, Wilson DJ, Rudel T, Wyllie DH, Fraunholz MJ. 2016. Natural mutations in a Staphylococcus aureus virulence regulator attenuate cytotoxicity but permit bacteremia and abscess formation. Proceedings of the National Academy of Sciences. DOI: 10.1073/pnas. 1520255113.

Delauné A, Dubrac S, Blanchet C, Poupel O, Mäder U, Hiron A, Leduc A, Fitting C, Nicolas P, Cavaillon J-M, Adib-Conquy M, Msadek T. 2012. The WalKR system controls major staphylococcal virulence genes and is involved in triggering the host inflammatory response. Infection and immunity 80:3438-3453. DOI: 10.1128/IAI.00195-12. 
603 Earle SG, Wu C-H, Charlesworth J, Stoesser N, Claire Gordon N, Walker TM, Spencer CCA,

604

605

606

607

608

609

610

611

612

613

614

615

616

617

618

619

620

621

622

623

624

625

626

627

628

629

630

631

632

633

634

635

636

637

638

639

640

641

642 Iqbal Z, Clifton DA, Hopkins KL, Woodford N, Grace Smith E, Ismail N, Llewelyn MJ, Peto TE, Crook DW, McVean G, Sarah Walker A, Wilson DJ. 2016. Identifying lineage effects when controlling for population structure improves power in bacterial association studies. Nature Microbiology 1:16041. DOI: 10.1038/nmicrobiol.2016.41.

Fey PD, Endres JL, Yajjala VK, Widhelm TJ, Boissy RJ, Bose JL, Bayles KW. 2013. A genetic resource for rapid and comprehensive phenotype screening of nonessential Staphylococcus aureus genes. mBio 4:e0537-12. DOI: 10.1128/mBio.00537-12.

Fowler VG Jr, Sakoulas G, McIntyre LM, Meka VG, Arbeit RD, Cabell CH, Stryjewski ME, Eliopoulos GM, Reller LB, Corey GR, Jones T, Lucindo N, Yeaman MR, Bayer AS. 2004. Persistent bacteremia due to methicillin-resistant Staphylococcus aureus infection is associated with agr dysfunction and low-level in vitro resistance to thrombin-induced platelet microbicidal protein. The Journal of infectious diseases 190:1140-1149. DOI: $10.1086 / 423145$.

Frees D, Qazi SNA, Hill PJ, Ingmer H. 2003. Alternative roles of ClpX and ClpP in Staphylococcus aureus stress tolerance and virulence. Molecular microbiology 48:15651578 .

Friedman JH. 2001. Greedy function approximation: a gradient boosting machine. Annals of statistics:1189-1232.

Gagnaire J, Dauwalder O, Boisset S, Khau D, Freydière A-M, Ader F, Bes M, Lina G, Tristan A, Reverdy M-E, Marchand A, Geissmann T, Benito Y, Durand G, Charrier J-P, Etienne J, Welker M, Van Belkum A, Vandenesch F. 2012. Detection of Staphylococcus aureus deltatoxin production by whole-cell MALDI-TOF mass spectrometry. PloS one 7:e40660. DOI: 10.1371/journal.pone.0040660.

Genestier A-L, Michallet M-C, Prévost G, Bellot G, Chalabreysse L, Peyrol S, Thivolet F, Etienne J, Lina G, Vallette FM, Vandenesch F, Genestier L. 2005. Staphylococcus aureus Panton-Valentine leukocidin directly targets mitochondria and induces Bax-independent apoptosis of human neutrophils. The Journal of clinical investigation 115:3117-3127. DOI: 10.1172/JCI22684.

Grosser MR, Paluscio E, Thurlow LR, Dillon MM, Cooper VS, Kawula TH, Richardson AR. 2018. Genetic requirements for Staphylococcus aureus nitric oxide resistance and virulence. PLoS pathogens 14:e1006907. DOI: 10.1371/journal.ppat.1006907.

Grumann D, Nübel U, Bröker BM. 2014. Staphylococcus aureus toxins--their functions and genetics. Infection, genetics and evolution: journal of molecular epidemiology and evolutionary genetics in infectious diseases 21:583-592. DOI: 10.1016/j.meegid.2013.03.013.

Hardy BL, Dickey SW, Plaut RD, Riggins DP, Stibitz S, Otto M, Merrell DS. 2019. Corynebacterium pseudodiphtheriticum Exploits Staphylococcus aureus Virulence Components in a Novel Polymicrobial Defense Strategy. mBio 10. DOI: 10.1128/mBio.02491-18.

Peer) reviewing PDF | (2019:10:41844:2:1:NEW 4 Feb 2020) 
643 Inouye M, Dashnow H, Raven L-A, Schultz M, Pope B, Tomita T, Zobel J, Holt K. 2014. 644 SRST2: Rapid genomic surveillance for public health and hospital microbiology labs.

645 Genome Med. 6:90. DOI: 10.1186/s13073-014-0090-6.

646 Jacquet R, LaBauve AE, Akoolo L, Patel S, Alqarzaee AA, Wong Fok Lung T, Poorey K, 647 Stinear TP, Thomas VC, Meagher R, Parker D. 2019. Dual gene expression analysis

648 identifies factors associated with Staphylococcus aureus virulence in diabetic mice. Infection 649 and immunity. DOI: 10.1128/IAI.00163-19.

650 Jaillard M, Lima L, Tournoud M, Mahé P, van Belkum A, Lacroix V, Jacob L. 2018. A fast and 651 agnostic method for bacterial genome-wide association studies: bridging the gap between 652 kmers and genetic events. bioRxiv:297754. DOI: 10.1101/297754.

653

654

655

656

657

658

659

660

661

662

663

664

665

666

667

668

669

670

671

672

673

674

675

676

677

678

679

680

681

682

Jain DS, Gupte SR, Aduri R. 2018. A Data Driven Model for Predicting RNA-Protein Interactions based on Gradient Boosting Machine. Scientific reports 8:9552. DOI: 10.1038/s41598-018-27814-2.

Janzon L, Löfdahl S, Arvidson S. 1989. Identification and nucleotide sequence of the delta-lysin gene, hld, adjacent to the accessory gene regulator (agr) of Staphylococcus aureus. Molecular \& general genetics: MGG 219:480-485. DOI: 10.1007/bf00259623.

Jarraud S, Mougel C, Thioulouse J, Lina G, Meugnier H, Forey F, Nesme X, Etienne J, Vandenesch F. 2002. Relationships between Staphylococcus aureus genetic background, virulence factors, agr groups (alleles), and human disease. Infection and immunity 70:631641.

Jolley KA, Bray JE, Maiden MCJ. 2018. Open-access bacterial population genomics: BIGSdb software, the PubMLST.org website and their applications. Wellcome open research 3:124. DOI: 10.12688/wellcomeopenres.14826.1.

Kasimir S, Schönfeld W, Alouf JE, König W. 1990. Effect of Staphylococcus aureus delta-toxin on human granulocyte functions and platelet-activating-factor metabolism. Infection and immunity 58:1653-1659.

Kim D-W, Park J-Y, Park K-D, Kim T-H, Lee W-J, Lee S-J, Kim J. 2009. Are there predominant strains and toxins of Staphylococcus aureus in atopic dermatitis patients?

Genotypic characterization and toxin determination of S. aureus isolated in adolescent and adult patients with atopic dermatitis. The Journal of dermatology 36:75-81. DOI: 10.1111/j.1346-8138.2009.00592.x.

Kitur K, Parker D, Nieto P, Ahn DS, Cohen TS, Chung S, Wachtel S, Bueno S, Prince A. 2015. Toxin-Induced Necroptosis Is a Major Mechanism of Staphylococcus aureus Lung Damage. PLoS pathogens 11:e1004820. DOI: 10.1371/journal.ppat.1004820.

Laabei M, Recker M, Rudkin JK, Aldeljawi M, Gulay Z, Sloan TJ, Williams P, Endres JL, Bayles KW, Fey PD, Yajjala VK, Widhelm T, Hawkins E, Lewis K, Parfett S, Scowen L, Peacock SJ, Holden M, Wilson D, Read TD, van den Elsen J, Priest NK, Feil EJ, Hurst LD, Josefsson E, Massey RC. 2014. Predicting the virulence of MRSA from its genome sequence. Genome research 24:839-849. DOI: 10.1101/gr.165415.113.

Laabei M, Uhlemann A-C, Lowy FD, Austin ED, Yokoyama M, Ouadi K, Feil E, Thorpe HA,

Peer) reviewing PDF | (2019:10:41844:2:1:NEW 4 Feb 2020) 
683

684

685

686

687

688

689

690

691

692

693

694

695

696

697

698

699

700

701

702

703

704

705

706

707

708

709

710

711

712

713

714

715

716

717

718

719

720

721

722

Williams B, Perkins M, Peacock SJ, Clarke SR, Dordel J, Holden M, Votintseva AA, Bowden R, Crook DW, Young BC, Wilson DJ, Recker M, Massey RC. 2015. Evolutionary Trade-Offs Underlie the Multi-faceted Virulence of Staphylococcus aureus. PLoS biology 13:e1002229. DOI: 10.1371/journal.pbio.1002229.

Lees JA, Vehkala M, Välimäki N, Harris SR, Chewapreecha C, Croucher NJ, Marttinen P, Davies MR, Steer AC, Tong SYC, Honkela A, Parkhill J, Bentley SD, Corander J. 2016. Sequence element enrichment analysis to determine the genetic basis of bacterial phenotypes. Nature communications 7:12797. DOI: 10.1038/ncomms12797.

Li T, He L, Song Y, Villaruz AE, Joo H-S, Liu Q, Zhu Y, Wang Y, Qin J, Otto M, Li M. 2015. AraC-Type Regulator Rsp Adapts Staphylococcus aureus Gene Expression to Acute Infection. Infection and immunity 84:723-734. DOI: 10.1128/IAI.01088-15.

McKevitt AI, Bjornson GL, Mauracher CA, Scheifele DW. 1990. Amino acid sequence of a deltalike toxin from Staphylococcus epidermidis. Infection and immunity 58:1473-1475.

Mittal P, Brindle J, Stephen J, Plotkin JB, Kudla G. 2018. Codon usage influences fitness through RNA toxicity. Proceedings of the National Academy of Sciences of the United States of America:201810022. DOI: 10.1073/pnas.1810022115.

Monk IR, Shah IM, Xu M, Tan M-W, Foster TJ. 2012. Transforming the untransformable: application of direct transformation to manipulate genetically Staphylococcus aureus and Staphylococcus epidermidis. mBio 3. DOI: 10.1128/mBio.00277-11.

Monk IR, Tree JJ, Howden BP, Stinear TP, Foster TJ. 2015. Complete Bypass of Restriction Systems for Major Staphylococcus aureus Lineages. mBio 6:e00308-15. DOI: 10.1128/mBio.00308-15.

Nakamura Y, Oscherwitz J, Cease KB, Chan SM, Muñoz-Planillo R, Hasegawa M, Villaruz AE, Cheung GYC, McGavin MJ, Travers JB, Otto M, Inohara N, Núñez G. 2013.

Staphylococcus $\delta$-toxin induces allergic skin disease by activating mast cells. Nature 503:397-401. DOI: 10.1038/nature12655.

Nguyen M, Brettin T, Long SW, Musser JM, Olsen RJ, Olson R, Shukla M, Stevens RL, Xia F, Yoo H, Davis JJ. 2018. Developing an in silico minimum inhibitory concentration panel test for Klebsiella pneumoniae. Scientific reports 8:421. DOI: 10.1038/s41598-017-18972-w.

Nguyen M, Long SW, McDermott PF, Olsen RJ, Olson R, Stevens RL, Tyson GH, Zhao S, Davis JJ. 2019. Using Machine Learning To Predict Antimicrobial MICs and Associated Genomic Features for Nontyphoidal Salmonella. Journal of clinical microbiology 57. DOI: 10.1128/JCM.01260-18.

Novick RP. 2003. Autoinduction and signal transduction in the regulation of staphylococcal virulence. Molecular microbiology 48:1429-1449.

Ondov BD, Treangen TJ, Melsted P, Mallonee AB, Bergman NH, Koren S, Phillippy AM. 2016. Mash: fast genome and metagenome distance estimation using MinHash. Genome biology 17:132. DOI: 10.1186/s13059-016-0997-x.

Otto M. 2013. Community-associated MRSA: what makes them special? International journal of medical microbiology: IJMM 303:324-330. DOI: 10.1016/j.ijmm.2013.02.007. 
723

724

725

726

727

728

729

730

731

732

733

734

735

736

737

738

739

740

741

742

743

744

745

746

747

748

749

750

751

752

753

754

755

756

757

758

759

760

761

762

Otto M. 2014. Staphylococcus aureus toxins. Current opinion in microbiology 17:32-37. DOI: 10.1016/j.mib.2013.11.004.

Page AJ, Cummins CA, Hunt M, Wong VK, Reuter S, Holden MTG, Fookes M, Falush D, Keane JA, Parkhill J. 2015. Roary: rapid large-scale prokaryote pan genome analysis.

Bioinformatics 31:3691-3693. DOI: 10.1093/bioinformatics/btv421.

Pagel M. 1999. Inferring the historical patterns of biological evolution. Nature 401:877-884. DOI: $10.1038 / 44766$.

Page AJ, Taylor B, Delaney AJ, Soares J, Seemann T, Keane JA, Harris SR. 2016. SNP-sites: rapid efficient extraction of SNPs from multi-FASTA alignments. Microbial genomics 2:e000056. DOI: 10.1099/mgen.0.000056.

Penley M, Morran L. 2018. Assessment of Caenorhabditis elegans Competitive Fitness in the Presence of a Bacterial Parasite. BIO-PROTOCOL 8. DOI: 10.21769/bioprotoc. 2971.

Peschel A, Otto M. 2013. Phenol-soluble modulins and staphylococcal infection. Nature reviews. Microbiology 11:667-673. DOI: 10.1038/nrmicro3110.

Petit RA 3rd, Read TD. 2018. Staphylococcus aureus viewed from the perspective of 40,000+ genomes. PeerJ 6:e5261. DOI: 10.7717/peerj.5261.

Power RA, Parkhill J, de Oliveira T. 2016. Microbial genome-wide association studies: lessons from human GWAS. Nature reviews. Genetics. DOI: 10.1038/nrg.2016.132.

Priest NK, Rudkin JK, Feil EJ, van den Elsen JMH, Cheung A, Peacock SJ, Laabei M, Lucks DA, Recker M, Massey RC. 2012. From genotype to phenotype: can systems biology be used to predict Staphylococcus aureus virulence? Nature reviews. Microbiology 10:791797. DOI: $10.1038 /$ nrmicro2880.

Pustišek N, Vurnek Živković M, Šitum M. 2016. Quality of Life in Families with Children with Atopic Dermatitis. Pediatric dermatology 33:28-32. DOI: 10.1111/pde.12698.

Qiang X, Chen H, Ye X, Su R, Wei L. 2018. M6AMRFS: Robust Prediction of N6Methyladenosine Sites With Sequence-Based Features in Multiple Species. Frontiers in genetics 9:495. DOI: 10.3389/fgene.2018.00495.

Quave CL, Horswill AR. 2018. Identification of Staphylococcal Quorum Sensing Inhibitors by Quantification of õ-Hemolysin with High Performance Liquid Chromatography. In: Leoni L, Rampioni G eds. Quorum Sensing: Methods and Protocols. New York, NY: Springer New York, 363-370. DOI: 10.1007/978-1-4939-7309-5_27.

Quave CL, Plano LRW, Bennett BC. 2011. Quorum sensing inhibitors of Staphylococcus aureus from Italian medicinal plants. Planta medica 77:188-195. DOI: 10.1055/s-0030-1250145.

Quinlan AR, Hall IM. 2010. BEDTools: a flexible suite of utilities for comparing genomic features. Bioinformatics 26:841-842. DOI: 10.1093/bioinformatics/btq033.

R Core Team, R Foundation for Statistical Computing, Vienna, Austria. 2016.R: A language and environment for statistical computing

Read TD, Massey RC. 2014. Characterizing the genetic basis of bacterial phenotypes using genome-wide association studies: a new direction for bacteriology. Genome medicine 6:109. DOI: 10.1186/s13073-014-0109-z. 
763

764

765

766

767

768

769

770

771

772

773

774

775

776

777

778

779

780

781

782

783

784

785

786

787

788

789

790

791

792

793

794

795

796

797

798

799

800

801

802

Recker M, Laabei M, Toleman MS, Reuter S, Saunderson RB, Blane B, Török ME, Ouadi K, Stevens E, Yokoyama M, Steventon J, Thompson L, Milne G, Bayliss S, Bacon L, Peacock SJ, Massey RC. 2017. Clonal differences in Staphylococcus aureus bacteraemia-associated mortality. Nature microbiology 2:1381-1388. DOI: 10.1038/s41564-017-0001-x.

Rose HR, Holzman RS, Altman DR, Smyth DS, Wasserman GA, Kafer JM, Wible M, Mendes RE, Torres VJ, Shopsin B. 2015. Cytotoxic Virulence Predicts Mortality in Nosocomial Pneumonia Due to Methicillin-Resistant Staphylococcus aureus. The Journal of infectious diseases 211:1862-1874. DOI: 10.1093/infdis/jiu554.

Rudkin JK, Edwards AM, Bowden MG, Brown EL, Pozzi C, Waters EM, Chan WC, Williams P, O'gara JP, Massey RC. 2012. Methicillin resistance reduces the virulence of healthcareassociated methicillin-resistant Staphylococcus aureus by interfering with the agr quorum sensing system. The Journal of infectious diseases 205:798-806.

Rudkin JK, McLoughlin RM, Preston A, Massey RC. 2017. Bacterial toxins: Offensive, defensive, or something else altogether? PLoS pathogens 13:e1006452. DOI: 10.1371/journal.ppat.1006452.

Sakoulas G, Eliopoulos GM, Fowler VG Jr, Moellering RC Jr, Novick RP, Lucindo N, Yeaman MR, Bayer AS. 2005. Reduced susceptibility of Staphylococcus aureus to vancomycin and platelet microbicidal protein correlates with defective autolysis and loss of accessory gene regulator (agr) function. Antimicrobial agents and chemotherapy 49:2687-2692. DOI: 10.1128/AAC.49.7.2687-2692.2005.

Sanchez-Garcia R, Sorzano COS, Carazo JM, Segura J. 2019. BIPSPI: a method for the prediction of partner-specific protein-protein interfaces. Bioinformatics 35:470-477. DOI: 10.1093/bioinformatics/bty647.

Schneider CA, Rasband WS, Eliceiri KW. 2012. NIH Image to ImageJ: 25 years of image analysis. Nature methods 9:671-675.

Seemann T. 2014. Prokka: rapid prokaryotic genome annotation. Bioinformatics 30:2068-2069. DOI: 10.1093/bioinformatics/btu153.

Solís-Lemus C, Bastide P, Ané C. 2017. PhyloNetworks: A Package for Phylogenetic Networks. Molecular biology and evolution 34:3292-3298. DOI: 10.1093/molbev/msx235.

Somerville GA, Cockayne A, Dürr M, Peschel A, Otto M, Musser JM. 2003. Synthesis and deformylation of Staphylococcus aureus delta-toxin are linked to tricarboxylic acid cycle activity. Journal of bacteriology 185:6686-6694.

Soong G, Paulino F, Wachtel S, Parker D, Wickersham M, Zhang D, Brown A, Lauren C, Dowd M, West E, Horst B, Planet P, Prince A. 2015. Methicillin-Resistant Staphylococcus aureus Adaptation to Human Keratinocytes. mBio 6. DOI: 10.1128/mBio.00289-15.

Stamatakis A. 2014. RAxML version 8: a tool for phylogenetic analysis and post-analysis of large phylogenies. Bioinformatics 30:1312-1313. DOI: 10.1093/bioinformatics/btu033. Tange O. 2018. GNU Parallel 2018. Lulu.com.

Walsh IM, Bowman MA, Clark PL. 2019. Synonymous codon substitutions perturb cotranslational protein folding and significantly impair cell fitness. bioRxiv:666552. DOI: 
803

804

805

806

807

808

809

810

811

812

813

814

815

816

817

818

819

820

821

822

823

824

825

826

$10.1101 / 666552$.

Wang R, Braughton KR, Kretschmer D, Bach T-HL, Queck SY, Li M, Kennedy AD, Dorward DW, Klebanoff SJ, Peschel A, DeLeo FR, Otto M. 2007. Identification of novel cytolytic peptides as key virulence determinants for community-associated MRSA. Nature medicine 13:1510-1514. DOI: 10.1038/nm1656.

Wang H, Liu C, Deng L. 2018. Enhanced Prediction of Hot Spots at Protein-Protein Interfaces Using Extreme Gradient Boosting. Scientific reports 8:14285. DOI: 10.1038/s41598-01832511-1.

Williams H, Flohr C. 2006. How epidemiology has challenged 3 prevailing concepts about atopic dermatitis. The Journal of allergy and clinical immunology 118:209-213. DOI: 10.1016/j.jaci.2006.04.043.

Xu SX, McCormick JK. 2012. Staphylococcal superantigens in colonization and disease. Frontiers in cellular and infection microbiology 2:52. DOI: 10.3389/fcimb.2012.00052.

Yeung M, Balma-Mena A, Shear N, Simor A, Pope E, Walsh S, McGavin MJ. 2011. Identification of major clonal complexes and toxin producing strains among Staphylococcus aureus associated with atopic dermatitis. Microbes and infection / Institut Pasteur 13:189197. DOI: 10.1016/j.micinf.2010.10.023.

Young BC, Wu C-H, Claire Gordon N, Cole K, Price JR, Liu E, Sheppard A, Perera S, Charlesworth J, Golubchik T, Iqbal Z, Bowden R, Massey R, Paul J, Crook DW, Peto TEA, Sarah Walker A, Llewelyn M, Wyllie DH, Wilson D. 2017. Severe infections emerge from the microbiome by adaptive evolution. bioRxiv:116681. DOI: $10.1101 / 116681$.

Zhou X, Stephens M. 2012. Genome-wide efficient mixed-model analysis for association studies. Nature genetics 44:821-824. DOI: 10.1038/ng.2310. 


\section{Figure 1}

Characterization of $\delta$-toxin production in 124 S. aureus strains.

Supernatants from 124 S. aureus strains from the Network on Antimicrobial Resistance in Staphylococcus aureus (NARSA) repository were subjected to high performance liquid chromatography (HPLC). The amount of $\delta$-toxin present in samples is proportional to the area under the peak generated by UV absorbance when passing through the HPLC detector. Production ranged from 0 to 97234 .

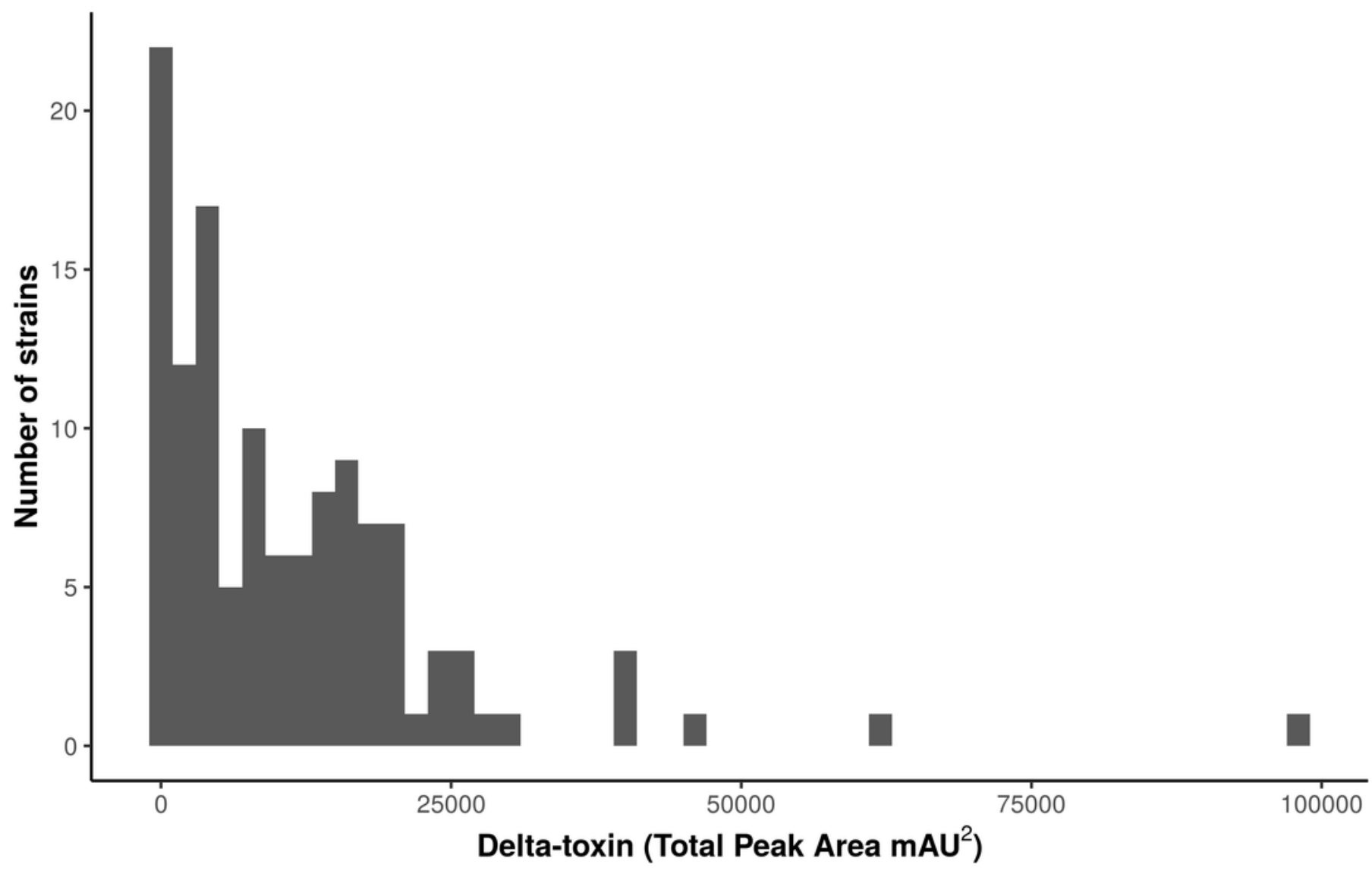


Figure 2

$\delta$-toxin production across the $S$. aureus phylogeny.

124 S. aureus strains in 23 clonal complexes (CC) were used to create a core genome phylogeny using RAxML. Clonal complexes are represented in the inner ring and color coded. Red bars represent $\delta$-toxin values from HPLC. Missing bars indicate $\delta$-toxin was undetectable by HPLC.

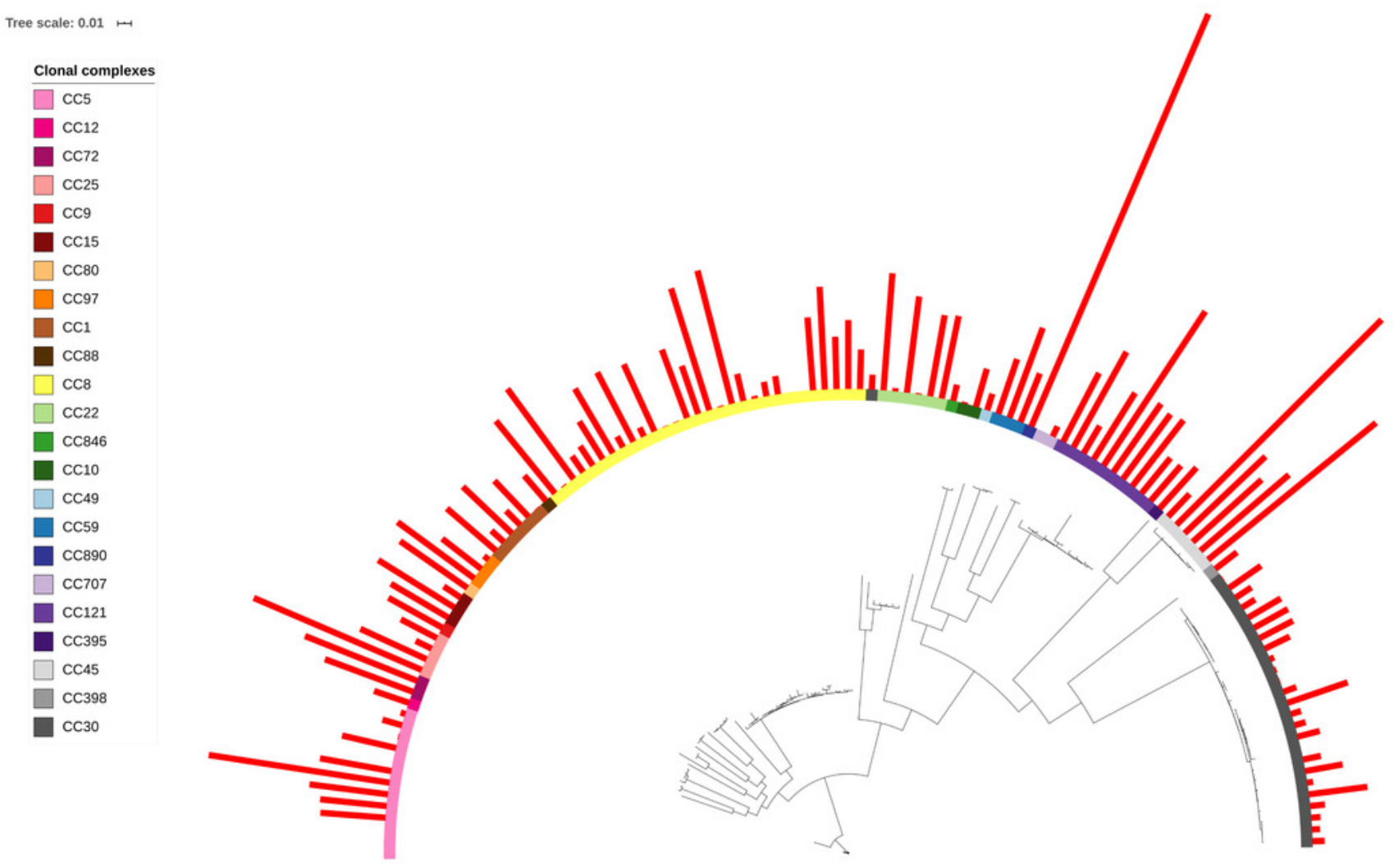


Figure 3

Associations of $\delta$-toxin production to methicillin resistance, agr type and clonal complex.

(A) Differences in $\delta$-toxin production between MSSA and MRSA strains. (B) Differences in $\delta$ toxin production between agr types. (C) Differences in $\delta$-toxin production between CCs. * $\mathrm{p}<.05, * * \mathrm{p}<.01, * * *<.001$ 
A

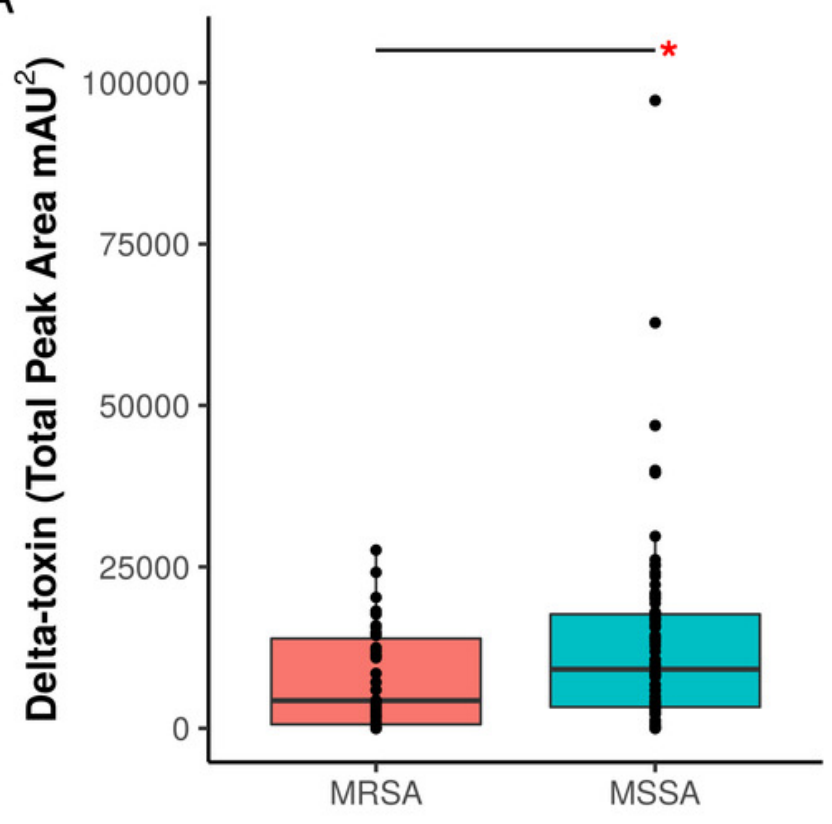

B

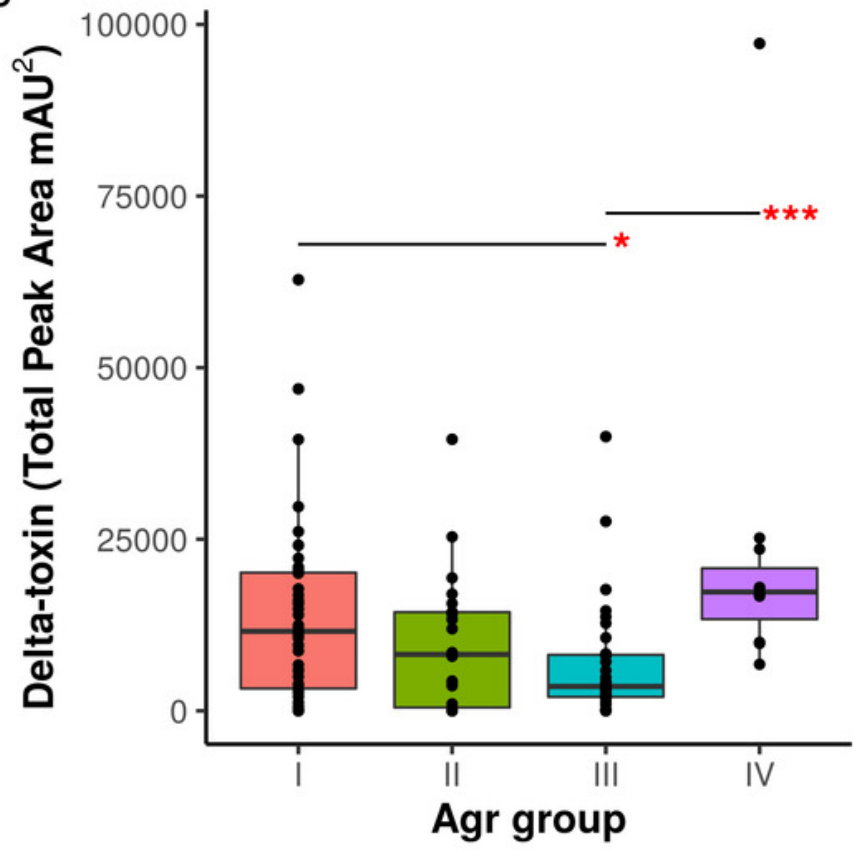

C

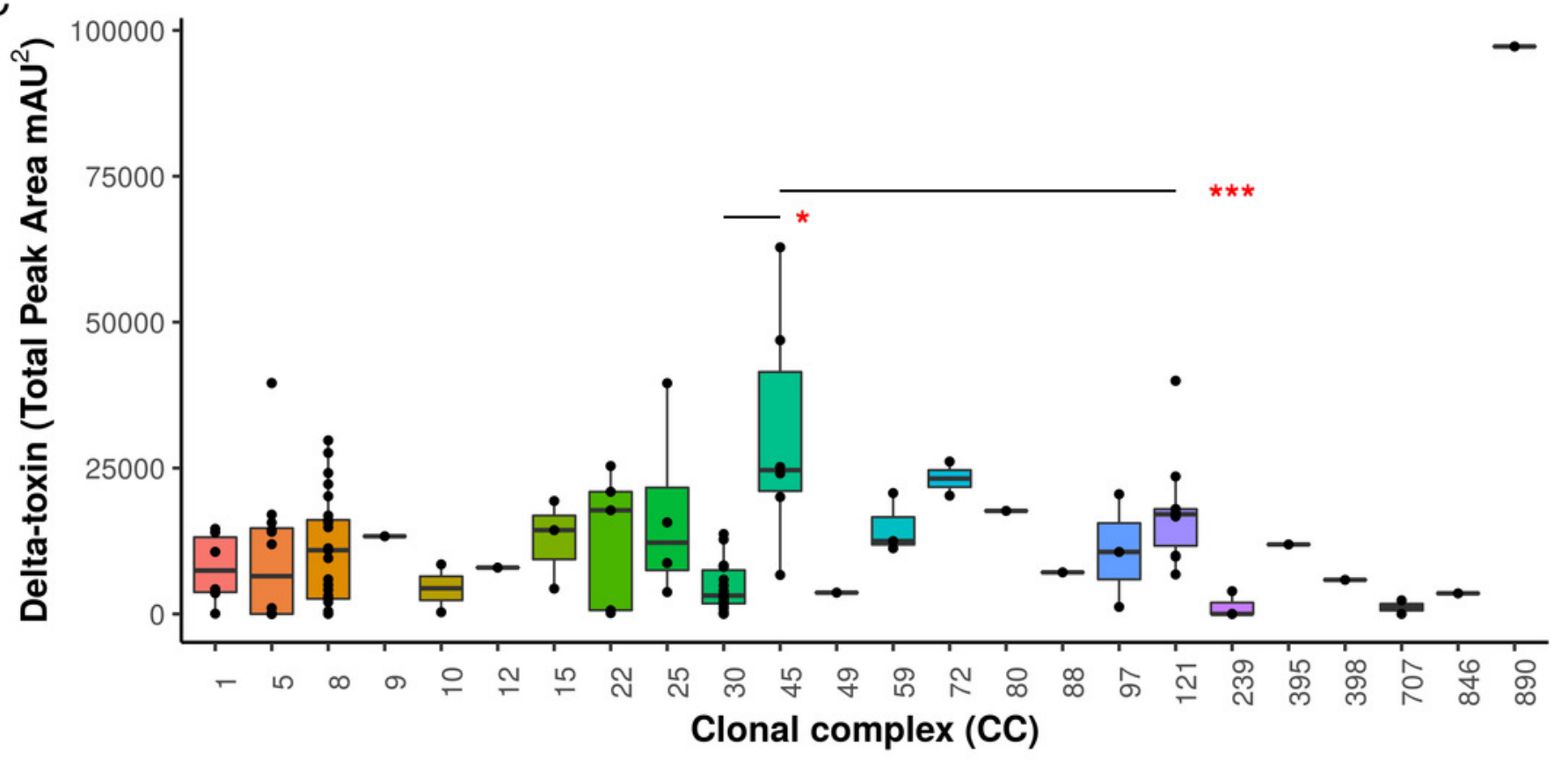


Figure 4

Impact of gene knockouts on $\delta$-toxin production.

A subset of genes that were found to be significantly associated with $\delta$-toxin by GWAS were tested for their effect of $\delta$-toxin production. $\delta$-toxin from transposon mutants from the Nebraska Transposon Mutant Library (NTML) was measured via HPLC. * $p<.05$

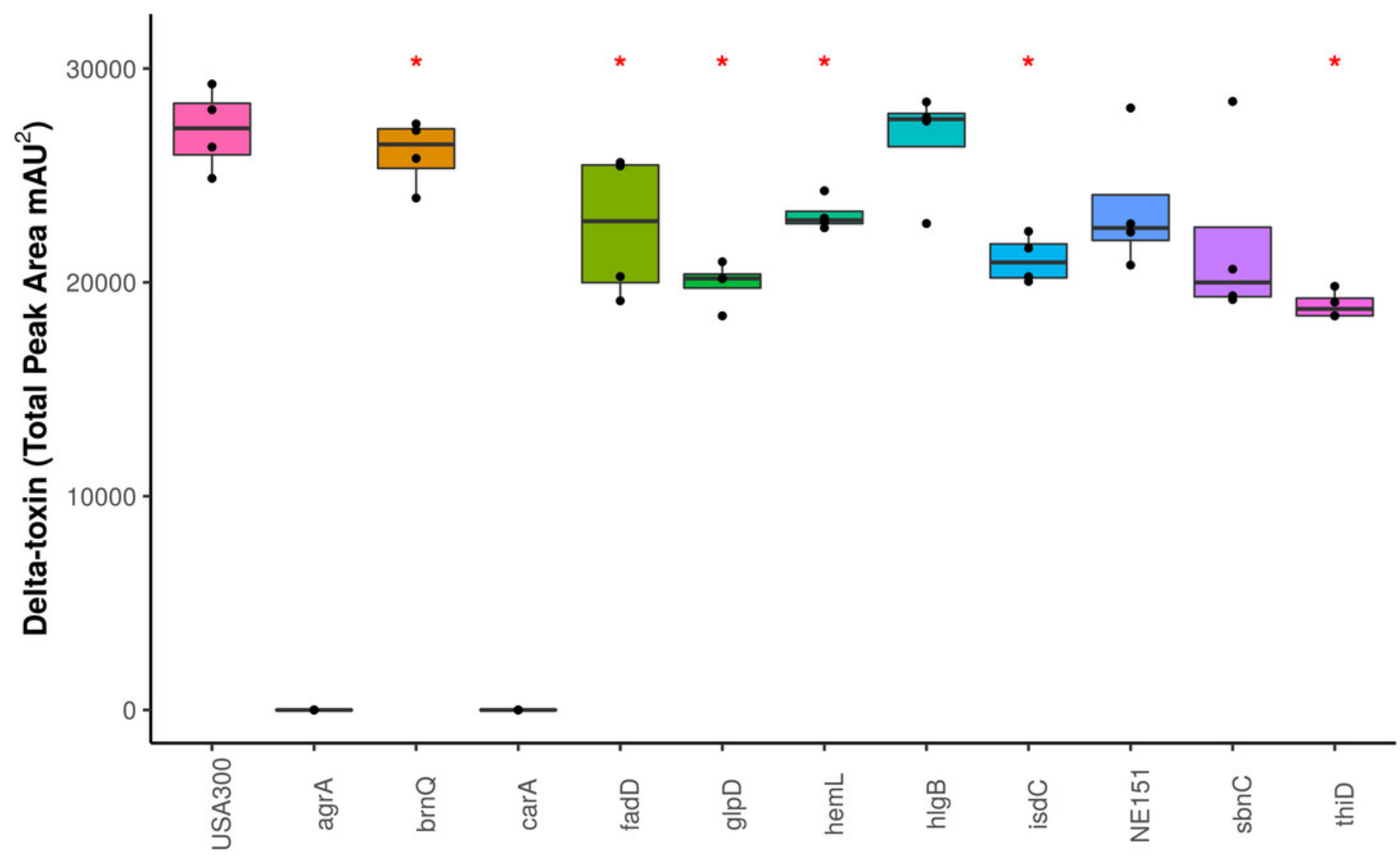




\section{Figure 5}

Impact of carA on hemolysis and virulence of $S$. aureus in C. elegans.

(A-E) S. aureus strains were spotted on $5 \%$ sheep's blood TSA II and incubated at $37{ }^{\circ} \mathrm{C}$ for 24 hours followed by 24 hours at $4{ }^{\circ} \mathrm{C}$. Representative colonies are being shown. (F) L4 C. elegans were fed either USA300 S. aureus (purple), a carA transposon mutant USA300 (red), a carA transposon mutant with vector (yellow), a carA transposon mutant complemented with the native USA300 carA allele, or a carA transposon mutant complemented with a carA allele that produces no $\delta$-toxin. Survival was scored at 24 and 48 hours. Survival counts are normalized against C. elegans fed on OP50 E. coli.
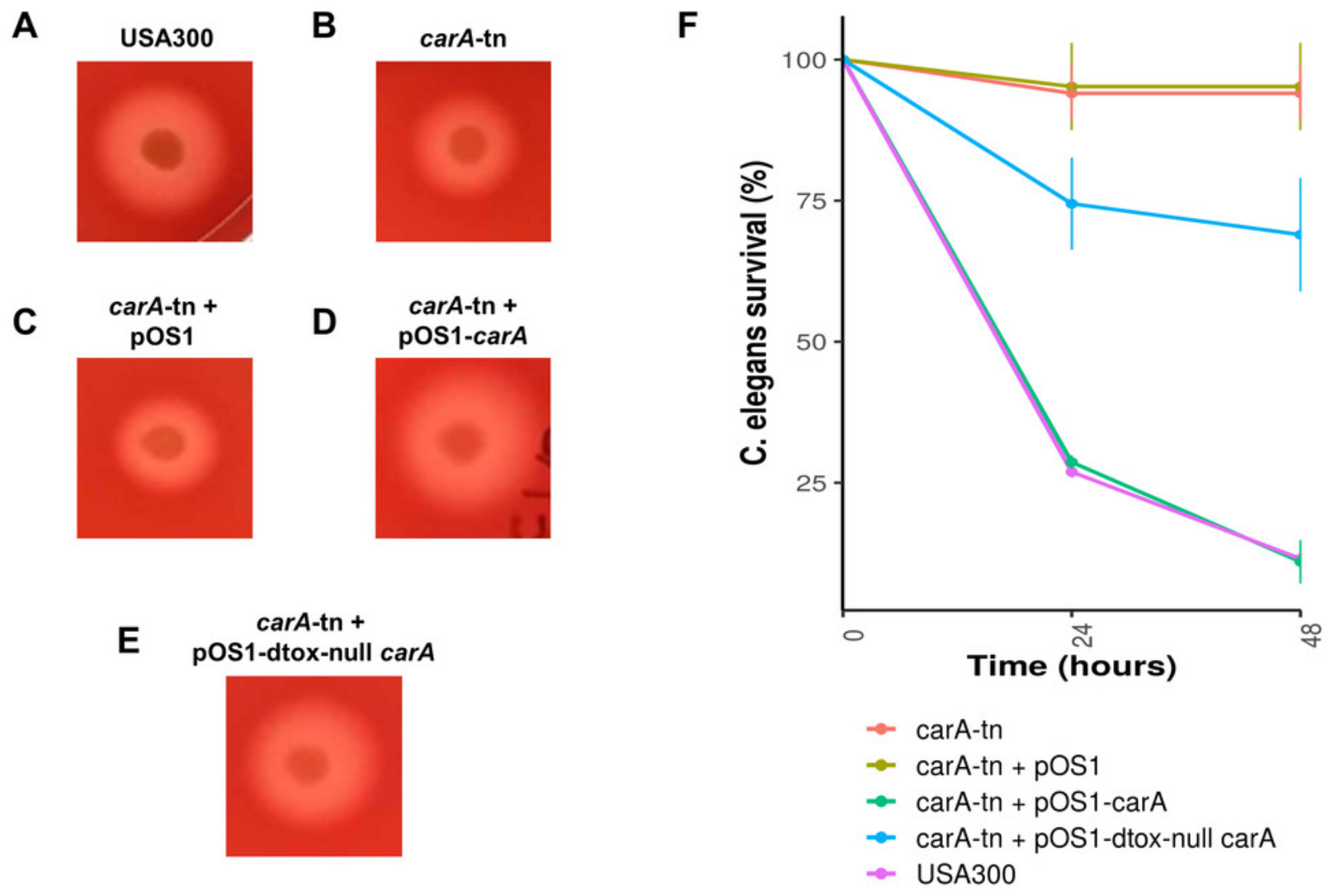


\section{Table $\mathbf{1}$ (on next page)}

XGBoost model performance

XGBoost models were trained with and without metadata (ST, CC, Agr group, and MSSA/MRSA status). 10-fold cross-validation was used to assess model performance. For all measures, the average performance across cross-validation is reported. For non-binary classification, individual precision and recall measures are weighted according to its proportion in the overall dataset during averaging, and Cohen's Kappa is calculated using squared weights. Specificity is not measured for non-binary classification models as it is included in the weighted precision and recall measures 
1

\begin{tabular}{|l|l|l|l|l|l|}
\hline Model & Precision & Recall & Specificity & $\begin{array}{l}\text { AUROC } \\
\mathbf{9 5 \%} \text { CI) }\end{array}$ & $\begin{array}{l}\text { Cohen's Kappa } \\
\text { (p-value) }\end{array}$ \\
\hline $\begin{array}{l}\text { Binary predictor }+/- \\
\text { metadata }\end{array}$ & .875 & .333 & .990 & $\begin{array}{l}.697 \\
(.553, .840)\end{array}$ & $.429(.000000037)$ \\
\hline $\begin{array}{l}\text { Four category } \\
\text { predictor }+ \text { metadata }\end{array}$ & $\begin{array}{l}.423 \\
\text { (weighted) }\end{array}$ & $\begin{array}{l}.443 \\
\text { (weighted) }\end{array}$ & N/A & $\begin{array}{l}.664 \\
(.597, .731)\end{array}$ & $\begin{array}{l}.255(.00441) \\
\text { (weighted) }\end{array}$ \\
\hline $\begin{array}{l}\text { Four category } \\
\text { predictor - metadata }\end{array}$ & $\begin{array}{l}.451 \\
\text { (weighted) }\end{array}$ & $\begin{array}{l}.326 \\
\text { (weighted) }\end{array}$ & N/A & $\begin{array}{l}.667 \\
(.576, .758)\end{array}$ & $\begin{array}{l}.133(.131) \\
\text { (weighted) }\end{array}$ \\
\hline
\end{tabular}

2 


\section{Table 2 (on next page)}

Characterization of hemolysis activity of USA300, carA mutant, and complemented strains.

S. aureus strains were spotted on $5 \%$ sheep's blood TSA II and incubated at $37^{\circ} \mathrm{C}$ for 24 hours followed by 24 hours at $4^{\circ} \mathrm{C}$. Plates were photographed using a lightbox and processed in Imagej. Hemolysis rings and colonies $(n=10)$ for each strain were measured using Imagej. 
1

\begin{tabular}{|l|l|l|}
\hline Strain & Average Hemolysis (mm) & Standard deviation (mm) \\
\hline USA300 & 9.0945 & .508 \\
\hline carA-tn & 5.6450 & .360 \\
\hline carA-tn + pOS1 & 5.0459 & .446 \\
\hline carA-tn + pOS1-carA & 7.3305 & .536 \\
\hline carA-tn + pOS1-dtox null carA & 7.0114 & .422 \\
\hline
\end{tabular}

2 